\title{
Independent associations of income and education with nutrient intakes in Brazilian adults: 2008-2009 National Dietary Survey
}

\author{
Marina Campos Araujo*, Eliseu Verly Junior, Washington Leite Junger and \\ Rosely Sichieri \\ Social Medicine Institute, Department of Epidemiology, State University of Rio de Janeiro, Rua São Francisco \\ Xavier 524, $7^{\circ}$ andar, sala E7002, Maracanã 20550-900, Rio de Janeiro, RJ, Brazil
}

Submitted 23 January 2013: Final revision received 10 September 2013: Accepted 18 September 2013: First published online 13 November 2013

\begin{abstract}
Objective: To verify associations of income and education with nutrient intakes in Brazilian adults.

Design: Data from the population-based National Dietary Survey conducted in 2008-2009. Family per capita income and education levels were categorized into quartiles. Prevalences of inadequate nutrient intakes and excessive intakes of saturated fat and $\mathrm{Na}$ were calculated by using the method prescribed by the National Cancer Institute. The Estimated Average Requirement was used as a reference for micronutrient intake. Linear regression models for both the independent and the mutually adjusted associations of education and income with nutrient intakes were tested. Interaction between education and income was tested.

Setting: Households ( $n$ 13569) selected using a two-stage cluster sampling design.

Subjects: Food records for two non-consecutive days were obtained for 21003 Brazilian adults (aged 20-59 years).

Results: For most of eleven nutrients, the prevalence of inadequate intake declined with increasing income and education levels; however, it remained high across all income and education quartiles. Excessive intake of saturated fat and low fibre intake increased with both variables. Most nutrients were independently associated with income and education in both sexes. Fe, vitamin $\mathrm{B}_{12}$ and $\mathrm{Na}$ intakes among women were associated only with education. There was an interaction between income and education for $\mathrm{Na}$ intake in men, $\mathrm{P}$ intake in women and $\mathrm{Ca}$ intake in both sexes.

Conclusions: Education is one important step to improve nutrient intakes in Brazil. Emphasis should be laid on enhancing dietary knowledge and formulating economic strategies that would allow lower-income individuals to adopt a healthy diet.
\end{abstract}

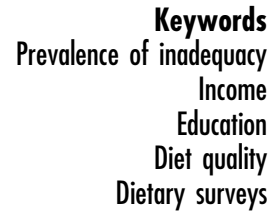

Many studies, conducted mainly in developed countries, demonstrate that consumption of better-quality diets characterized by greater intakes of fruits, fresh vegetables, whole grains and lean meats, higher micronutrient intakes, lower intakes of added sugars, fats and $\mathrm{Na}$, and lower energy density - are positively associated with socio-economic status (SES) ${ }^{(1-13)}$. Almost all studies used education and income as a proxy of SES.

Although the association between SES and diet quality is well established in developed nations, it is still understudied in middle-income countries like Brazil. Brazilian studies that focused on the relationship between SES and obesity showed an inverse association between obesity and income during the period from 1975 through $2003^{(14)}$ and reported that the influence of education on obesity prevalence was more pronounced than that of income, especially among women ${ }^{(15)}$.

In relation to diet, the hypothesis that education and income do not have the same impact on diet quality is supported by the fact that income and education reflect different aspects of $\mathrm{SES}^{(16,17)}$. For example, Monsivais and Drewnowski ${ }^{(4)}$ found that education was a stronger predictor of energy density than was household income in American adults. Among the French elderly, higher energy intake was associated with higher income, but not with education level ${ }^{(18)}$. A review study showed 
that education had a stronger effect on diet quality than did income ${ }^{(19)}$.

It may be worthwhile to test this hypothesis in Brazil since education and income are not highly correlated; people in the lowest education level vary in income. The explanation for this is that the recent increase of $28 \%$ in household income between 2004 and 2009 was not accompanied by a similar improvement in education status, with an increase of only 0.6 full years of study being observed in the same period ${ }^{(20)}$.

Findings of the Brazilian national study on nutritional deficiencies showed that the major problems among adults are the inadequacy of $\mathrm{Ca}, \mathrm{Mg}, \mathrm{P}, \mathrm{Na}$ and vitamins $\mathrm{A}$, $\mathrm{C}, \mathrm{D}$ and $\mathrm{E}$, for which the prevalence of inadequate intake was $\geq 60 \%$, and the inadequacy of $\mathrm{Zn}$, thiamin and $\mathrm{Fe}$ (only in women), for which the prevalence of inadequate intake was $\geq 20 \%$.

We aimed to study the independent association of income and education with those nutrients with a high prevalence of inadequacy among Brazilian adults. To our knowledge, the present study is the first to investigate the relationship between education and income and nutrient intakes in a nationwide sample in a middle-income country.

\section{Methods}

\section{Population}

The present study analysed data obtained from the Brazilian National Dietary Survey (NDS), which was carried out along with the 2008-2009 Household Budget Survey (HBS) conducted by the Brazilian Institute of Geography and Statistics. The NDS was the first national survey of individual dietary intake. The sample for the Brazilian HBS was selected using a two-stage cluster sampling design. In the first stage, the primary sampling units, census tracts, were selected by systematic sampling with probability proportional to the number of households. Census tracts were stratified to include representatives of all Brazilian regions, including both urban and rural areas, and also different socio-economic levels.
In the second stage, households were selected by simple random sampling. The 2008-2009 HBS included 68373 households from 4694 census tracts. A sub-sample of $25 \%$ of the HBS households was estimated for the NDS. Finally, a total of 13569 households agreed to participate in the survey (21\% were non-responders). Individuals aged 10 years or older ( $n$ 34003) living in the selected households were included in the dietary survey. For the present paper we included only individuals aged 20-59 years, with the exception of pregnant and lactating women ( $n$ 1065), yielding a final sample of 21003 Brazilian adults $^{(22)}$. A total of 166 adults who did not answer the education question were excluded from analysis.

The sample weights used represent the adult population as in the census for the five Brazilian regions (North, South, Midwest, Southeast and Northeast) and the urban or rural areas. The general characteristics of adults investigated in the NDS were very similar to those of adults from the HBS, which has representativeness for the Brazilian population (Table 1). The present study was approved by the local ethics committee (CAAE 0011.0.259.000-11).

\section{Dietary intake}

Food records of two non-consecutive days were used to estimate food intake. All respondents received a booklet, which contained explanations on how to fill out the records and pictures of tableware to help them estimate the amount of food consumed. Individuals were encouraged to fill out information on foods, beverages, amounts consumed and the time and place of meals. Trained interviewers were responsible for entering the data obtained during the household survey in a computer database while at the household. Details about the pretest, training and validation of the food records have been published elsewhere ${ }^{(22)}$.

The energy and nutrient intakes were estimated using a food composition table $e^{(23)}$ and a food portion table $e^{(24)}$ specially developed for the survey. Standard recipes were created and nutritional information was based on the Brazilian Table of Food Composition and the Nutrient

Table 1 General characteristics and prevalence of obesity among Brazilian adults aged 20-59 years (excluding pregnant and lactating women) from the 2008-2009 Household Budget Survey and National Dietary Survey

\begin{tabular}{|c|c|c|c|c|}
\hline & \multicolumn{2}{|c|}{$\begin{array}{c}\text { Household Budget Survey } \\
n 97916 \text { (sample size) } \\
n 100695789 \text { (expanded sample size) }\end{array}$} & \multicolumn{2}{|c|}{$\begin{array}{c}\text { National Dietary Survey } \\
n 21003 \text { (sample size) } \\
n 100286871 \text { (expanded sample size) }\end{array}$} \\
\hline & Mean or \% & SE & Mean or \% & SE \\
\hline Age (years) & $37 \cdot 5$ & 0.06 & $37 \cdot 5$ & $0 \cdot 1$ \\
\hline Education (years of education) & $8 \cdot 1$ & 0.04 & $8 \cdot 1$ & 0.06 \\
\hline Income (monthly household per capita) (\$US) & 396 & $6 \cdot 9$ & 399 & $10 \cdot 6$ \\
\hline BMI $\left(\mathrm{kg} / \mathrm{m}^{2}\right)$ & $25 \cdot 4$ & 0.03 & $25 \cdot 5$ & 0.06 \\
\hline Men (\%) & $50 \cdot 0$ & 0.2 & $50 \cdot 2$ & $0 \cdot 4$ \\
\hline Obesity $\left(\mathrm{BMI} \geq 30 \mathrm{~kg} / \mathrm{m}^{2}\right)(\%)$ & $14 \cdot 0$ & 0.2 & $14 \cdot 5$ & $0 \cdot 4$ \\
\hline
\end{tabular}

Values are presented as weighted means or percentages and their standard errors. 
Data System for Research, version 2008 (Nutrition Coordinating Center, University of Minnesota, Minneapolis, MN, USA). The nutrient intake data did not include the consumption of supplements and/or medications.

\section{Socio-economic status}

Socio-economic characteristics were recorded during the household interviews. Monthly household per capita income from all monetary and non-monetary sources of income, including gifts, donations, employment, self-employment and participation in cash transfer programmes, was calculated. The total household income was divided by the number of members in the household to calculate the per capita household income and then classified into quartiles: $\leq \$$ US $120 \cdot 70,>\$$ US $120 \cdot 70-227 \cdot 30,>$ SUS $227 \cdot 30-440 \cdot 90$ and $>$ \$US $440 \cdot 90$ per capita per month. The conversion rate at the time of the HBS ( $\$$ US $1=2 \cdot 38$ Brazilian Real) was applied to convert the amounts to US dollars. Moreover, the official minimum monthly wage in Brazil during this period was \$US $174 \cdot 40$.

Education was defined as the number of full years of study and was evaluated in quartiles according to the number of full years of education: $\leq 4$ years, $5-8$ years, 9-11 years and $>11$ years.

\section{Data analyses}

The prevalences of inadequate and excessive nutrient intakes and the usual nutrient intakes were estimated by using the National Cancer Institute (NCI) method. This method estimates the usual intake after adjusting for within-person variance, by considering the consumptionday amount using the linear mixed-effects model on a transformed scale ${ }^{(25)}$. To estimate the usual nutrient intake expressed as a ratio, such as energy from saturated fat, the NCI method was also used and the usual ratio of intake was considered ${ }^{(26)}$.

The NCI method assumes a simple random sampling. For a complex sample design, the BBR (balanced repeated replication) technique with the modification described by Fay ${ }^{(27)}$ is used to estimate standard errors and $P$ values. Fay's BRR method considers design situations in which there are two primary sampling units per stratum. Since the NDS had more than two primary sampling units per stratum, a method called GBHS (grouped balanced half samples) was used. This method randomly assigns primary sampling units into two groups of approximately the same size in each stratum ${ }^{(28,29)}$.

The values of Estimated Average Requirement (EAR) proposed by the US Institute of Medicine were used as the reference for micronutrient intake ${ }^{(30-35)}$, and the EAR cut-off point method was used to estimate the prevalence of inadequate nutrient intake ${ }^{(36)}$. The prevalence of inadequate nutrient intake represents the proportion of the population below the median requirement. Because the distribution of $\mathrm{Fe}$ requirement among women of reproductive age is skewed, the EAR cut-off point method could not be applied. In this case, the probability approach method was used to estimate the prevalence of inadequate $\mathrm{Fe}$ intake ${ }^{(33)}$.

The $\mathrm{Na}$ intake in the Brazilian population is excessively high $^{(37)}$; therefore, we used the Tolerable Upper Intake Level (UL) values, also proposed by the Institute of Medicine, to calculate the proportion of the population above the UL who were at risk of adverse effects ${ }^{(34)}$. Furthermore, the $\mathrm{Na}$ intake represents the total $\mathrm{Na}$ in food, taking into account both natural $\mathrm{Na}$ and added $\mathrm{Na}$.

The cut-off point for energy intake from saturated fat (7\%) was based on the recommendation of the Brazilian Society of Cardiology ${ }^{(38)}$. For dietary fibre intake, we adopted a value of $25 \mathrm{~g} / \mathrm{d}$ considering a diet of $8368 \mathrm{~kJ}$ (2000 kcal) as recommended by the Brazilian Ministry of Health $^{(39)}$. In the present paper, we considered $12.5 \mathrm{~g}$ dietary fibre $/ 4184 \mathrm{~kJ}(1000 \mathrm{kcal})$. For both $\mathrm{Na}$ intake and energy intake from saturated fat, the inadequacy was based on excessive consumption of these nutrients.

The prevalences of inadequate and excessive nutrient intakes and their standard errors were calculated according to quartiles of monthly household per capita income and education, and models to estimate the prevalence included the five Brazilian regions (North, South, Midwest, Southeast and Northeast) and the area in which the households were located (urban or rural) as covariates.

Linear regression models were used to evaluate the associations between nutrient intakes (dependent variable) and SES (independent variable). The individual usual energy and nutrient intakes were evaluated as continuous variables and income and education were assessed as quartiles. The models considered both the independent and the mutually adjusted associations of education and income with nutrient intakes, adjusted for age and additionally for energy intake for analysis of minerals and vitamins. Furthermore, the aged-adjusted weighted mean nutrient intakes by quartiles of income and education and means mutually adjusted according to sex were estimated, except for those analyses with a statistically significant interaction between education and income. The interaction terms between sex and socio-economic variables and between income and education were tested for all nutrients. For those nutrients with a statistically significant interaction, aged-adjusted weighted means according to both income and education quartiles and by sex were shown graphically.

Both prevalence of inadequacy and linear models analyses took into account the day-to-day intra-individual variation in nutrient intake. The MSM (Multiple Source Method) was used to estimate individual usual nutrient intakes in the linear models ${ }^{(40)}$.

All statistical analyses were weighted and performed using survey procedures from the statistical software package SAS release $9 \cdot 3$ to take into account the sample design effect. Weighting factors were corrected for non-response, thus allowing representative population estimates. 


\section{Results}

For seven out of the eleven nutrients analysed (Ca, P, $\mathrm{Zn}$, thiamin and vitamins $\mathrm{B}_{12}, \mathrm{~A}$ and $\mathrm{C}$ ), the prevalence of inadequate intake decreased with increasing income among men. In women, the prevalence of inadequate intake decreased with income for eight nutrients $(\mathrm{Ca}$, $\mathrm{P}, \mathrm{Fe}, \mathrm{Zn}$, thiamin and vitamins $\mathrm{B}_{12}, \mathrm{~A}$ and $\mathrm{C}$; Table 2). Similar results were observed for both sexes with regard to education levels (Table 3 ).

For men and women, the excessive intake of energy from saturated fat and inadequate fibre intake increased with income (Table 2) and education level (Table 3). The prevalence of excessive $\mathrm{Na}$ intake in both sexes, and the prevalence of inadequate $\mathrm{Fe}$ intake in men, did not change with income (Table 2) or education level (Table 3). Inadequate intakes of $\mathrm{Zn}$ among men and of $\mathrm{Fe}$ among women aged 21-59 years changed only with income (Table 2).

The interaction term between sex and income was statistically significant $(P<0 \cdot 05)$ only for fibre intake and the interaction between sex and education was statistically significant for fibre, $\mathrm{Fe}, \mathrm{Zn}$ and vitamin $\mathrm{B}_{12}$ intakes (data not shown). Once sex modified the effect of the association between some nutrients and SES variables, all analysis were stratified according to sex.

Most nutrients were independently associated with income and education, such as energy, energy from saturated fat, fibre, thiamin and vitamins $\mathrm{A}$ and $\mathrm{C}$ in both sexes (Tables 4 and 5) and also for $\mathrm{P}$ among men (Table 4). Fe, vitamin $\mathrm{B}_{12}$ and $\mathrm{Na}$ intakes among women were associated only with education (Table 5). However, Fe intake was associated only with income among men (Table 4). Vitamin $\mathrm{B}_{12}$ intake was not associated with income or education among men (Table 4). An intriguing result was verified for Zn intake in that it was positively associated with income and education in the mutually adjusted model in both sexes, despite no association being observed in the simple models for income and education with the exception of the statistically significant association between education and Zn intake among men.

The interaction between income and education was statistically significant $(P<0.05)$ for energy, Ca, P, Fe and $\mathrm{Na}$ intakes among men and for energy from saturated fat, $\mathrm{Ca}$ and $\mathrm{P}$ intakes among women. $\mathrm{Na}$ intake in men, $\mathrm{P}$ intake in women and $\mathrm{Ca}$ intake in both sexes were associated with income and education in the simple linear models; however, due to strong interaction between

Table 2 Sample size ( $n$ 21003), cut-off points used as guidelines and prevalences of inadequate nutrient intakes according to income quartiles $^{*}$ in Brazilian adults aged 20-59 years (excluding pregnant and lactating women), 2008-2009 National Dietary Survey

\begin{tabular}{|c|c|c|c|c|c|c|c|c|c|}
\hline \multirow[b]{2}{*}{ Nutrient } & \multirow[b]{2}{*}{ Cut-off point } & \multicolumn{2}{|c|}{ Q1 ( $n$ 6764) } & \multicolumn{2}{|c|}{ Q2 (n 5539) } & \multicolumn{2}{|c|}{ Q3 (n 4722) } & \multicolumn{2}{|c|}{ Q4 (n 3978) } \\
\hline & & $\%$ & $95 \% \mathrm{Cl}$ & $\%$ & $95 \% \mathrm{Cl}$ & $\%$ & $95 \% \mathrm{Cl}$ & $\%$ & $95 \% \mathrm{Cl}$ \\
\hline \multicolumn{10}{|l|}{ Men $(n$ 9974) } \\
\hline Energy from saturated fat & $>7 \%$ & 63 & 61,66 & 79 & 78,81 & 88 & 86,89 & 93 & 92,94 \\
\hline Dietary fibre & $<12.5 \mathrm{~g} / 4184 \mathrm{~kJ}$ & 59 & 56,63 & 65 & 61,69 & 73 & 68,78 & 82 & 79,85 \\
\hline $\mathrm{Ca}$ & $<800 \mathrm{mg}$ & 93 & 92,94 & 89 & 87,91 & 83 & 82,85 & 74 & 72,77 \\
\hline $\mathrm{P}$ & $<580 \mathrm{mg}$ & 9 & 8,11 & 6 & 5,8 & 5 & 4,6 & 4 & 3,4 \\
\hline Fet & - & 5 & - & 3 & - & 3 & - & 4 & - \\
\hline $\mathrm{Zn}$ & $<9.4 \mathrm{mg}$ & 32 & 30,35 & 23 & 20,26 & 21 & 18,24 & 23 & 21,24 \\
\hline Thiamin & $<1.0 \mathrm{mg}$ & 43 & 40,46 & 31 & 24,38 & 27 & 23,31 & 23 & 20,25 \\
\hline Vitamin $\mathrm{B}_{12}$ & $<2 \mu g$ & 8 & 6,10 & 7 & 5,8 & 5 & 4,7 & 4 & 2,5 \\
\hline Vitamin A & $<625 \mu \mathrm{g}$ & 92 & 90,94 & 86 & 84,89 & 81 & 79,82 & 71 & 63,79 \\
\hline Vitamin C & $<75 \mathrm{mg}$ & 59 & 56,61 & 53 & 50,57 & 42 & 40,44 & 35 & 33,37 \\
\hline $\mathrm{Na}$ & $>2300 \mathrm{mg}$ & 88 & 86,89 & 91 & 89,93 & 92 & 91,93 & 89 & 88,90 \\
\hline \multicolumn{10}{|l|}{ Women (n 11 029) } \\
\hline Energy from saturated fat & $>7 \%$ & 71 & 69,73 & 86 & 85,87 & 90 & 88,92 & 95 & 93,97 \\
\hline Dietary fibre & $<12.5 \mathrm{~g} / 4184 \mathrm{~kJ}$ & 65 & 62,67 & 71 & 68,73 & 76 & 74,77 & 79 & 76,82 \\
\hline $20-50$ years & $<800 \mathrm{mg}$ & 96 & 96,97 & 93 & 92,94 & 90 & 88,91 & 80 & 76,83 \\
\hline $51-59$ years & $<1000 \mathrm{mg}$ & 99 & 99,100 & 98 & 98,99 & 96 & 94,98 & 93 & 90,96 \\
\hline \multirow{2}{*}{\multicolumn{10}{|c|}{ Fet }} \\
\hline & & & & & & & & & \\
\hline 20-50 years & - & 38 & - & 31 & - & 29 & - & 29 & - \\
\hline $51-59$ years & - & 15 & - & 10 & - & 9 & - & 9 & - \\
\hline $\mathrm{Zn}$ & $<6.8 \mathrm{mg}$ & 29 & 27,31 & 20 & 18,23 & 17 & 16,19 & 17 & 16,19 \\
\hline Thiamin & $<0.9 \mathrm{mg}$ & 52 & 49,55 & 42 & 40,44 & 34 & 33,35 & 28 & 24,31 \\
\hline Vitamin $\mathrm{B}_{12}$ & $<2 \mu \mathrm{g}$ & 19 & 17,21 & 13 & 10,17 & 12 & 11,13 & 8 & 5,10 \\
\hline Vitamin A & $<500 \mu \mathrm{g}$ & 85 & 83,88 & 75 & 72,78 & 69 & 67,71 & 53 & 48,57 \\
\hline Vitamin C & $<60 \mathrm{mg}$ & 51 & 49,53 & 43 & 41,45 & 33 & 31,36 & 26 & 23,28 \\
\hline $\mathrm{Na}$ & $>2300 \mathrm{mg}$ & 63 & 62,65 & 69 & 67,72 & 71 & 69,73 & 70 & 67,73 \\
\hline
\end{tabular}

Values are presented as percentages and their $95 \%$ confidence intervals.

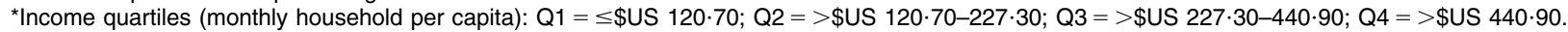
tCalculated by the probability approach method.

$\ddagger$ Calculated as retinol activity equivalents. 
Table 3 Sample size $(n 20837)^{\star}$, cut-off points used as guidelines and prevalences of inadequate nutrient intakes according to education quartilest in Brazilian adults aged 20-59 years (excluding pregnant and lactating women), 2008-2009 National Dietary Survey

\begin{tabular}{|c|c|c|c|c|c|c|c|c|c|}
\hline \multirow[b]{2}{*}{ Nutrient } & \multirow[b]{2}{*}{ Cut-off point } & \multicolumn{2}{|c|}{ Q1 (n 6672) } & \multicolumn{2}{|c|}{ Q2 (n 4911) } & \multicolumn{2}{|c|}{ Q3 (n 6526) } & \multicolumn{2}{|c|}{ Q4 (n 2728) } \\
\hline & & $\%$ & $95 \% \mathrm{Cl}$ & $\%$ & $95 \% \mathrm{Cl}$ & $\%$ & $95 \% \mathrm{Cl}$ & $\%$ & $95 \% \mathrm{Cl}$ \\
\hline \multicolumn{10}{|l|}{ Men ( $n$ 9893) } \\
\hline Energy from saturated fat & $>7 \%$ & 68 & 65,71 & 80 & 78,81 & 87 & 86,88 & 93 & 91,95 \\
\hline Dietary fibre & $<12.5 \mathrm{~g} / 4184 \mathrm{~kJ}$ & 55 & 49,60 & 67 & 63,72 & 79 & 76,81 & 82 & 81,84 \\
\hline $\mathrm{Ca}$ & $<800 \mathrm{mg}$ & 91 & 90,92 & 89 & 87,91 & 82 & 79,85 & 71 & 67,76 \\
\hline $\mathrm{P}$ & $<580 \mathrm{mg}$ & 8 & 7,9 & 7 & 6,8 & 4 & 3,6 & 3 & 2,5 \\
\hline Fe $\ddagger$ & - & 4 & - & 4 & - & 4 & - & 4 & - \\
\hline $\mathrm{Zn}$ & $<9.4 \mathrm{mg}$ & 26 & 24,28 & 25 & 23,27 & 23 & 18,27 & 25 & 22,28 \\
\hline Thiamin & $<1.0 \mathrm{mg}$ & 41 & 38,44 & 33 & 27,38 & 24 & 21,27 & 21 & 18,24 \\
\hline Vitamin $\mathrm{B}_{12}$ & $<2 \mu \mathrm{g}$ & 7 & 6,9 & 7 & 6,8 & 5 & 4,6 & 4 & 1,7 \\
\hline Vitamin A§ & $<625 \mu \mathrm{g}$ & 90 & 88,92 & 86 & 85,88 & 78 & 73,83 & 70 & 61,79 \\
\hline Vitamin C & $<75 \mathrm{mg}$ & 56 & 54,59 & 53 & 45,61 & 42 & 38,45 & 31 & 28,34 \\
\hline $\mathrm{Na}$ & $>2300 \mathrm{mg}$ & 89 & 88,91 & 90 & 89,91 & 92 & 90,94 & 87 & 84,91 \\
\hline \multicolumn{10}{|l|}{ Women ( $n$ 10944) } \\
\hline Energy from saturated fat & $>7 \%$ & 73 & 71,75 & 86 & 84,87 & 90 & 88,91 & 94 & 92,97 \\
\hline Dietary fibre & $<12 \cdot 5 \mathrm{~g} / 4184 \mathrm{~kJ}$ & 60 & 58,62 & 71 & 68,74 & 78 & 77,80 & 80 & 78,83 \\
\hline \multicolumn{10}{|l|}{$\mathrm{Ca}$} \\
\hline 20-50 years & $<800 \mathrm{mg}$ & 96 & 95,97 & 94 & 93,95 & 90 & 88,92 & 78 & 72,84 \\
\hline $51-59$ years & $<1000 \mathrm{mg}$ & 98 & 98,99 & 96 & 94,98 & 94 & 92,96 & 89 & 83,95 \\
\hline $\mathrm{P}$ & $<580 \mathrm{mg}$ & 25 & 23,27 & 20 & 18,21 & 15 & 13,16 & 8 & 4,11 \\
\hline \multicolumn{10}{|l|}{ Fe‡ } \\
\hline 20-50 years & - & 37 & - & 32 & - & 31 & - & 29 & - \\
\hline $51-59$ years & - & 9 & - & 11 & - & 9 & - & 10 & - \\
\hline $\mathrm{Zn}$ & $<6.8 \mathrm{mg}$ & 25 & 23,27 & 22 & 20,24 & 19 & 17,21 & 16 & 14,18 \\
\hline Thiamin & $<0.9 \mathrm{mg}$ & 52 & 50,53 & 42 & 40,44 & 33 & 30,35 & 26 & 20,33 \\
\hline Vitamin $\mathrm{B}_{12}$ & $<2 \mu \mathrm{g}$ & 20 & 17,22 & 14 & 12,15 & 11 & 8,13 & 7 & 5,9 \\
\hline Vitamin A§ & $<500 \mu \mathrm{g}$ & 84 & 82,86 & 75 & 73,77 & 66 & 64,69 & 51 & 43,59 \\
\hline Vitamin C & $<60 \mathrm{mg}$ & 50 & 47,54 & 43 & 40,46 & 33 & 32,35 & 24 & 20,28 \\
\hline $\mathrm{Na}$ & $>2300 \mathrm{mg}$ & 66 & 63,68 & 67 & 65,68 & 71 & 69,73 & 70 & 67,73 \\
\hline
\end{tabular}

Values are presented as percentages and their $95 \%$ confidence intervals.

*One hundred and sixty-six adults were excluded because they had no information about education.

tEducation quartiles (years of education): $Q 1=\leq 4$ years; $Q 2=5-8$ years; $Q 3=9-11$ years; $Q 4=>11$ years.

¥Calculated by the probability approach method.

$\S$ Calculated as retinol activity equivalents.

income and education, only the interaction term was statistically significant in the mutually adjusted model (Tables 4 and 5). Ca intake among men increased with income and education; however, this increase with income among men in the highest education quartile was not constant. In addition, Ca intake among women increased with income and education, although this increase with income was more evident in the highest education quartile. Similar results were observed for $\mathrm{P}$ intake among women. On the other hand, a negative association between income and $\mathrm{Na}$ intake was observed only among men in the highest educational level (Fig. 1).

\section{Discussion}

The present study used data from the first Brazilian NDS and revealed that inadequate nutrient intakes decreased with both income and education, with the exception of energy intake from saturated fat and intake of dietary fibre, for which there was a positive association. In addition, we found that most nutrients were associated with both income and education, despite that only educational level had an important role on the consumption of $\mathrm{Fe}$, vitamin $\mathrm{B}_{12}$ and $\mathrm{Na}$ among women and only income status was relevant for Fe consumption among men.

In accordance with the findings of our study, many studies have identified that food and nutrient intakes were healthier among wealthy people and those with higher education levels $^{(1-13)}$. On the other hand, our study showed that three markers of an unhealthy diet (higher energy intake, higher energy intake from saturated fat, lower fibre intake) were more evident in the high socio-economic groups. Our results indicate that even Brazilian people with high levels of education and resources to purchase and choose better food options do not necessarily achieve better-quality diets. A possible explanation is that the low-income population in Brazil still consumes traditional foods $^{(41)}$ and has lesser opportunity to consume food in places other than their homes ${ }^{(42)}$. Eating out of home has gained importance in diets worldwide and also in Brazil, and energy and saturated fat intakes increase with food eaten out of home, whereas fibre intake is reduced ${ }^{(42)}$.

Although there are few studies in middle- and lowerincome countries about the association between diet quality and SES variables, Tseng and Fang ${ }^{(43)}$ found similar results among Chinese immigrant women in the USA. They observed that higher education was significantly associated 
Table 4 Aged-adjusted weighted mean nutrient intakes by quartiles of income and education and mean nutrient intakes mutually adjusted among Brazilian men ( $n$ 9974) aged 20-59 years, 2008-2009 National Dietary Survey

\begin{tabular}{|c|c|c|c|c|c|c|c|c|c|}
\hline & \multirow[b]{2}{*}{ SES quartile* } & \multicolumn{2}{|c|}{ Income ( $n$ 9974) } & \multicolumn{2}{|c|}{ Education ( $n$ 9893)† } & \multicolumn{3}{|c|}{$\begin{array}{l}\text { Income adjusted for } \\
\text { education }(n \text { 9893)† }\end{array}$} & \multirow{2}{*}{$\begin{array}{c}\begin{array}{c}\text { Interaction between income } \\
\text { and education }\end{array} \\
P \\
\end{array}$} \\
\hline & & Mean & $P$ & Mean & $P$ & Mean $\ddagger$ & $P_{\text {Income }}$ & $P_{\text {Education }}$ & \\
\hline Energy (kJ)§ & $\begin{array}{l}\text { Q1 } \\
\text { Q2 } \\
\text { Q3 } \\
\text { Q4 } \\
\text { Diff** }\end{array}$ & $\begin{array}{c}8379 \\
8885 \\
9115 \\
9138 \\
9 \%\end{array}$ & $<0 \cdot 01$ & $\begin{array}{c}8703 \\
8754 \\
9117 \\
8942 \\
3 \%\end{array}$ & $0 \cdot 02$ & $\begin{array}{l}- \\
- \\
- \\
- \\
-\end{array}$ & $<0.01$ & $<0.01$ & $<0.01$ \\
\hline Energy from saturated fat (\%) & $\begin{array}{l}\text { Q1 } \\
\text { Q2 } \\
\text { Q3 } \\
\text { Q4 } \\
\text { Diff* }\end{array}$ & $\begin{array}{r}8 \cdot 2 \\
8 \cdot 9 \\
9 \cdot 5 \\
10 \cdot 0 \\
20 \%\end{array}$ & $<0.01$ & $\begin{array}{r}8 \cdot 3 \\
9 \cdot 0 \\
9 \cdot 5 \\
10 \cdot 2 \\
21 \%\end{array}$ & $<0 \cdot 01$ & $\begin{array}{c}8 \cdot 3 \\
9 \cdot 0 \\
9 \cdot 4 \\
9 \cdot 7 \\
15 \%\end{array}$ & $<0 \cdot 01$ & $<0.01$ & $0 \cdot 36$ \\
\hline Dietary fibre (g/4184 kJ) & $\begin{array}{c}\text { Q1 } \\
\text { Q2 } \\
\text { Q3 } \\
\text { Q4 } \\
\text { Diff* }^{\star *}\end{array}$ & $\begin{array}{c}11 \cdot 8 \\
11 \cdot 4 \\
10 \cdot 7 \\
9 \cdot 9 \\
-17 \%\end{array}$ & $<0 \cdot 01$ & $\begin{array}{c}12 \cdot 1 \\
11 \cdot 1 \\
10 \cdot 3 \\
9 \cdot 8 \\
-21 \%\end{array}$ & $<0.01$ & $\begin{array}{l}11 \cdot 6 \\
11 \cdot 3 \\
10 \cdot 8 \\
10 \cdot 4 \\
-11 \%\end{array}$ & $<0.01$ & $<0.01$ & 0.07 \\
\hline $\mathrm{Ca}(\mathrm{mg}) \|$ & $\begin{array}{c}\text { Q1 } \\
\text { Q2 } \\
\text { Q3 } \\
\text { Q4 } \\
\text { Diff** }^{*}\end{array}$ & $\begin{array}{c}489 \\
503 \\
548 \\
622 \\
25 \%\end{array}$ & $<0.01$ & $\begin{array}{c}493 \\
511 \\
558 \\
652 \\
30 \%\end{array}$ & $<0.01$ & $\begin{array}{l}- \\
- \\
- \\
- \\
-\end{array}$ & $0 \cdot 3$ & 0.06 & $<0.01$ \\
\hline$P(m g) \|$ & $\begin{array}{l}\text { Q1 } \\
\text { Q2 } \\
\text { Q3 } \\
\text { Q4 } \\
\text { Diff** }\end{array}$ & $\begin{array}{c}1080 \\
1059 \\
1079 \\
1130 \\
5 \%\end{array}$ & $<0.01$ & $\begin{array}{c}1078 \\
1062 \\
1084 \\
1157 \\
7 \%\end{array}$ & $<0.01$ & $\begin{array}{l}- \\
- \\
- \\
- \\
-\end{array}$ & $<0.01$ & $<0.01$ & $<0.01$ \\
\hline $\mathrm{Fe}(\mathrm{mg}) \|$ & $\begin{array}{l}\text { Q1 } \\
\text { Q2 } \\
\text { Q3 } \\
\text { Q4 } \\
\text { Diff** }\end{array}$ & $\begin{array}{l}13 \cdot 1 \\
13 \cdot 6 \\
13 \cdot 4 \\
12 \cdot 7 \\
-3 \%\end{array}$ & $<0.01$ & $\begin{array}{l}13 \cdot 7 \\
13 \cdot 3 \\
12 \cdot 9 \\
12 \cdot 6 \\
-8 \%\end{array}$ & $<0 \cdot 01$ & $\begin{array}{l}- \\
- \\
- \\
- \\
-\end{array}$ & 0.02 & 0.97 & 0.01 \\
\hline $\mathrm{Zn}(\mathrm{mg}) \|$ & $\begin{array}{c}\text { Q1 } \\
\text { Q2 } \\
\text { Q3 } \\
\text { Q4 } \\
\text { Diff** }\end{array}$ & $\begin{array}{c}12 \cdot 6 \\
12 \cdot 9 \\
12 \cdot 9 \\
12 \cdot 6 \\
0\end{array}$ & 0.9 & $\begin{array}{l}13 \cdot 0 \\
12 \cdot 9 \\
12 \cdot 5 \\
12 \cdot 6 \\
-3 \%\end{array}$ & $<0.01$ & $\begin{array}{r}12 \cdot 5 \\
12 \cdot 8 \\
12 \cdot 9 \\
12 \cdot 8 \\
2 \%\end{array}$ & 0.03 & $<0.01$ & 0.59 \\
\hline
\end{tabular}




\begin{tabular}{|c|c|c|c|c|c|c|c|c|c|}
\hline & \multirow[b]{2}{*}{ SES quartile* } & \multicolumn{2}{|c|}{ Income ( $n$ 9974) } & \multicolumn{2}{|c|}{ Education ( $n$ 9893)† } & \multicolumn{3}{|c|}{$\begin{array}{l}\text { Income adjusted for } \\
\text { education ( } n \text { 9893)t }\end{array}$} & \multirow{2}{*}{$\begin{array}{c}\text { Interaction between income } \\
\text { and education }\end{array}$} \\
\hline & & Mean & $P$ & Mean & $P$ & Mean‡ & $P_{\text {Income }}$ & $P_{\text {Education }}$ & \\
\hline Thiamin (mg)\| & $\begin{array}{l}\text { Q1 } \\
\text { Q2 } \\
\text { Q3 } \\
\text { Q4 } \\
\text { Diff** }\end{array}$ & $\begin{array}{l}1 \cdot 23 \\
1 \cdot 27 \\
1 \cdot 29 \\
1 \cdot 34 \\
9 \%\end{array}$ & $<0.01$ & $\begin{array}{r}1 \cdot 23 \\
1 \cdot 27 \\
1 \cdot 30 \\
1 \cdot 38 \\
12 \%\end{array}$ & $<0.01$ & $\begin{array}{l}1 \cdot 25 \\
1 \cdot 27 \\
1.28 \\
1.31 \\
5 \%\end{array}$ & $<0.01$ & $<0.01$ & $0 \cdot 62$ \\
\hline Vitamin $B_{12}(\mu \mathrm{g}) \|$ & $\begin{array}{l}\text { Q1 } \\
\text { Q2 } \\
\text { Q3 } \\
\text { Q4 } \\
\text { Diff** }^{*}\end{array}$ & $\begin{array}{l}5 \cdot 6 \\
5 \cdot 4 \\
5 \cdot 4 \\
5 \cdot 6 \\
0\end{array}$ & $0 \cdot 8$ & $\begin{array}{l}5 \cdot 6 \\
5 \cdot 3 \\
5 \cdot 4 \\
5 \cdot 6 \\
0\end{array}$ & 0.9 & $\begin{array}{l}5 \cdot 6 \\
5 \cdot 4 \\
5 \cdot 4 \\
5 \cdot 6 \\
0\end{array}$ & $0 \cdot 8$ & $0 \cdot 9$ & $0 \cdot 15$ \\
\hline Vitamin A $(\mu \mathrm{g}) \|, \boldsymbol{\oplus}$ & $\begin{array}{c}\text { Q1 } \\
\text { Q2 } \\
\text { Q3 } \\
\text { Q4 } \\
\text { Diff** }\end{array}$ & $\begin{array}{l}402 \\
430 \\
453 \\
519 \\
26 \%\end{array}$ & $<0.01$ & $\begin{array}{l}402 \\
423 \\
479 \\
532 \\
29 \%\end{array}$ & $<0.01$ & $\begin{array}{l}416 \\
435 \\
446 \\
487 \\
16 \%\end{array}$ & $<0.01$ & $<0.01$ & $0 \cdot 32$ \\
\hline Vitamin C (mg)\| & $\begin{array}{l}\text { Q1 } \\
\text { Q2 } \\
\text { Q3 } \\
\text { Q4 } \\
\text { Diff** }\end{array}$ & $\begin{array}{r}138 \\
147 \\
172 \\
189 \\
32 \%\end{array}$ & $<0.01$ & $\begin{array}{l}135 \\
152 \\
176 \\
203 \\
42 \%\end{array}$ & $<0.01$ & $\begin{array}{c}147 \\
150 \\
169 \\
172 \\
16 \%\end{array}$ & $<0.01$ & $<0.01$ & 0.9 \\
\hline $\mathrm{Na}(\mathrm{mg}) \|$ & $\begin{array}{l}\text { Q1 } \\
\text { Q2 } \\
\text { Q3 } \\
\text { Q4 } \\
\text { Diff** }\end{array}$ & $\begin{array}{l}3635 \\
3649 \\
3621 \\
3480 \\
-4 \%\end{array}$ & $<0.01$ & $\begin{array}{l}3651 \\
3626 \\
3581 \\
3472 \\
-5 \%\end{array}$ & $<0.01$ & $\begin{array}{l}- \\
- \\
- \\
- \\
-\end{array}$ & $0 \cdot 2$ & 0.06 & $<0.01$ \\
\hline
\end{tabular}

SES, socio-economic status

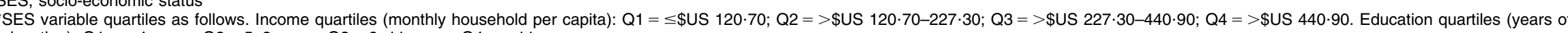
education): $\mathrm{Q} 1=\leq 4$ years; $\mathrm{Q} 2=5-8$ years; $\mathrm{Q} 3=9-11$ years; $\mathrm{Q} 4=>11$ years.

tEighty-one men were excluded because they had no information about education.

¥Mean nutrient intake by income and education quartles is shown in Fig. 1 for interaction term $P<0.05$

$\$ 1 \mathrm{~kJ}=0.239 \mathrm{kcal}$.

IIFurther adjusted for energy intake as a continuous variable by a linear model.

- Calculated as retinol activity equivalents. 
Table 5 Aged-adjusted weighted mean nutrient intakes by quartiles of income and education and mean nutrient intakes mutually adjusted among Brazilian among women ( $n$ 11029) aged 20-59 years (excluding pregnant and lactating women), 2008-2009 National Dietary Survey

\begin{tabular}{|c|c|c|c|c|c|c|c|c|c|}
\hline & \multirow[b]{2}{*}{ SES quartile* } & \multicolumn{2}{|c|}{ Income (n 11029) } & \multicolumn{2}{|c|}{ Education ( $n$ 10944)† } & \multicolumn{3}{|c|}{$\begin{array}{l}\text { Income adjusted for } \\
\text { education }(n \text { 10944)† }\end{array}$} & \multirow{2}{*}{$\begin{array}{c}\begin{array}{c}\text { Interaction between income } \\
\text { and education }\end{array} \\
P\end{array}$} \\
\hline & & Mean & $P$ & Mean & $P$ & Mean‡ & $P_{\text {Income }}$ & $P_{\text {Education }}$ & \\
\hline Energy $(\mathrm{kJ}) \S$ & $\begin{array}{c}\text { Q1 } \\
\text { Q2 } \\
\text { Q3 } \\
\text { Q4 } \\
\text { Diff* }^{*}\end{array}$ & $\begin{array}{l}6804 \\
7110 \\
7382 \\
7511 \\
10 \%\end{array}$ & $<0.01$ & $\begin{array}{l}6870 \\
7079 \\
7352 \\
7562 \\
10 \%\end{array}$ & $<0.01$ & $\begin{array}{c}6883 \\
7149 \\
7345 \\
7385 \\
7 \%\end{array}$ & $<0.01$ & $<0.01$ & $0 \cdot 07$ \\
\hline Energy from saturated fat (\%) & $\begin{array}{l}\text { Q1 } \\
\text { Q2 } \\
\text { Q3 } \\
\text { Q4 } \\
\text { Diff* }\end{array}$ & $\begin{array}{c}8 \cdot 7 \\
9 \cdot 6 \\
9 \cdot 9 \\
10 \cdot 5 \\
19 \%\end{array}$ & $<0.01$ & $\begin{array}{r}8 \cdot 8 \\
9 \cdot 6 \\
9 \cdot 9 \\
10 \cdot 5 \\
18 \%\end{array}$ & $<0 \cdot 01$ & $\begin{array}{l}- \\
- \\
- \\
- \\
-\end{array}$ & $<0.01$ & $<0.01$ & $<0.01$ \\
\hline Dietary fibre (g/4184 kJ) & $\begin{array}{l}\text { Q1 } \\
\text { Q2 } \\
\text { Q3 } \\
\text { Q4 } \\
\text { Diff* }^{\star *}\end{array}$ & $\begin{array}{l}11 \cdot 5 \\
10 \cdot 9 \\
10 \cdot 5 \\
10 \cdot 1 \\
-13 \%\end{array}$ & $<0 \cdot 01$ & $\begin{array}{l}11 \cdot 6 \\
10 \cdot 9 \\
10 \cdot 4 \\
10 \cdot 1 \\
-14 \%\end{array}$ & $<0 \cdot 01$ & $\begin{array}{l}11 \cdot 3 \\
10 \cdot 9 \\
10 \cdot 6 \\
10 \cdot 4 \\
-8 \%\end{array}$ & $<0.01$ & $<0.01$ & $0 \cdot 15$ \\
\hline $\mathrm{Ca}(\mathrm{mg}) \|$ & $\begin{array}{l}\text { Q1 } \\
\text { Q2 } \\
\text { Q3 } \\
\text { Q4 } \\
\text { Diff** }\end{array}$ & $\begin{array}{l}423 \\
451 \\
485 \\
558 \\
28 \%\end{array}$ & $<0.01$ & $\begin{array}{r}429 \\
455 \\
486 \\
569 \\
26 \%\end{array}$ & $<0.01$ & $\begin{array}{l}- \\
- \\
- \\
- \\
-\end{array}$ & $0 \cdot 07$ & $0 \cdot 7$ & $<0.01$ \\
\hline$P(m g) \|$ & $\begin{array}{l}\text { Q1 } \\
\text { Q2 } \\
\text { Q3 } \\
\text { Q4 } \\
\text { Diff** }\end{array}$ & $\begin{array}{l}873 \\
875 \\
888 \\
953 \\
9 \%\end{array}$ & $<0.01$ & $\begin{array}{c}872 \\
877 \\
899 \\
961 \\
10 \%\end{array}$ & $<0.01$ & $\begin{array}{l}- \\
- \\
- \\
- \\
-\end{array}$ & $0 \cdot 2$ & 0.09 & $<0.01$ \\
\hline $\mathrm{Fe}(\mathrm{mg}) \|$ & $\begin{array}{l}\text { Q1 } \\
\text { Q2 } \\
\text { Q3 } \\
\text { Q4 } \\
\text { Diff** }\end{array}$ & $\begin{array}{c}10 \cdot 1 \\
10 \cdot 4 \\
10 \cdot 3 \\
10 \cdot 1 \\
0\end{array}$ & $0 \cdot 6$ & $\begin{array}{l}10 \cdot 5 \\
10 \cdot 3 \\
10 \cdot 2 \\
10 \cdot 1 \\
-4 \%\end{array}$ & $<0.01$ & $\begin{array}{r}10 \cdot 1 \\
10 \cdot 4 \\
10 \cdot 4 \\
10 \cdot 2 \\
1 \%\end{array}$ & $0 \cdot 2$ & $<0.01$ & $0 \cdot 46$ \\
\hline $\mathrm{Zn}(\mathrm{mg}) \|$ & $\begin{array}{l}\text { Q1 } \\
\text { Q2 } \\
\text { Q3 } \\
\text { Q4 } \\
\text { Diff** }\end{array}$ & $\begin{array}{r}10 \cdot 0 \\
10 \cdot 3 \\
10 \cdot 3 \\
10 \cdot 2 \\
2 \%\end{array}$ & $0 \cdot 22$ & $\begin{array}{l}10 \cdot 4 \\
10 \cdot 3 \\
10 \cdot 1 \\
10 \cdot 2 \\
-2 \%\end{array}$ & 0.09 & $\begin{array}{r}9 \cdot 9 \\
10 \cdot 3 \\
10 \cdot 3 \\
10 \cdot 4 \\
5 \%\end{array}$ & $<0.01$ & $<0.01$ & $0 \cdot 18$ \\
\hline
\end{tabular}




\begin{tabular}{|c|c|c|c|c|c|c|c|c|c|}
\hline & \multirow[b]{2}{*}{ SES quartile* } & \multicolumn{2}{|c|}{ Income (n 11029) } & \multicolumn{2}{|c|}{ Education ( $n$ 10944)t } & \multicolumn{3}{|c|}{$\begin{array}{l}\text { Income adjusted for } \\
\text { education }(n \text { 10 944)† }\end{array}$} & \multirow{2}{*}{$\begin{array}{c}\begin{array}{c}\text { Interaction between income } \\
\text { and education }\end{array} \\
P\end{array}$} \\
\hline & & Mean & $P$ & Mean & $P$ & Mean‡ & $P_{\text {Income }}$ & $P_{\text {Education }}$ & \\
\hline Thiamin (mg)\| & $\begin{array}{l}\text { Q1 } \\
\text { Q2 } \\
\text { Q3 } \\
\text { Q4 } \\
\text { Diff** }\end{array}$ & $\begin{array}{l}1 \cdot 03 \\
1 \cdot 06 \\
1 \cdot 09 \\
1 \cdot 12 \\
8 \%\end{array}$ & $<0.01$ & $\begin{array}{l}1 \cdot 03 \\
1 \cdot 07 \\
1 \cdot 10 \\
1 \cdot 14 \\
10 \%\end{array}$ & $<0.01$ & $\begin{array}{l}1 \cdot 04 \\
1 \cdot 07 \\
1 \cdot 08 \\
1 \cdot 12 \\
7 \%\end{array}$ & $<0.01$ & $<0.01$ & 0.56 \\
\hline Vitamin $B_{12}(\mu \mathrm{g}) \|$ & $\begin{array}{l}\text { Q1 } \\
\text { Q2 } \\
\text { Q3 } \\
\text { Q4 } \\
\text { Diff** }\end{array}$ & $\begin{array}{l}4 \cdot 6 \\
4 \cdot 7 \\
4 \cdot 5 \\
4 \cdot 8 \\
4 \%\end{array}$ & $0 \cdot 4$ & $\begin{array}{l}4 \cdot 4 \\
4 \cdot 6 \\
4 \cdot 8 \\
4 \cdot 8 \\
9 \%\end{array}$ & $<0.01$ & $\begin{array}{c}4 \cdot 7 \\
4 \cdot 8 \\
4 \cdot 5 \\
4 \cdot 6 \\
-2 \%\end{array}$ & $0 \cdot 2$ & $<0.01$ & $0 \cdot 47$ \\
\hline Vitamin A $(\mu \mathrm{g}) \|, \uparrow$ & $\begin{array}{l}\text { Q1 } \\
\text { Q2 } \\
\text { Q3 } \\
\text { Q4 } \\
\text { Diff* }\end{array}$ & $\begin{array}{c}389 \\
441 \\
426 \\
516 \\
29 \%\end{array}$ & $<0.01$ & $\begin{array}{c}369 \\
429 \\
465 \\
525 \\
35 \%\end{array}$ & $<0.01$ & $\begin{array}{l}411 \\
449 \\
415 \\
478 \\
15 \%\end{array}$ & $<0.01$ & $<0.01$ & 0.58 \\
\hline Vitamin C (mg)\| & $\begin{array}{l}\text { Q1 } \\
\text { Q2 } \\
\text { Q3 } \\
\text { Q4 } \\
\text { Diff** }\end{array}$ & $\begin{array}{l}135 \\
147 \\
177 \\
180 \\
28 \%\end{array}$ & $<0.01$ & $\begin{array}{l}132 \\
143 \\
171 \\
194 \\
39 \%\end{array}$ & $<0.01$ & $\begin{array}{l}144 \\
150 \\
170 \\
165 \\
13 \%\end{array}$ & $<0.01$ & $<0.01$ & $0 \cdot 8$ \\
\hline $\mathrm{Na}(\mathrm{mg})$ & $\begin{array}{l}\text { Q1 } \\
\text { Q2 } \\
\text { Q3 } \\
\text { Q4 } \\
\text { Diff** }^{4}\end{array}$ & $\begin{array}{l}2892 \\
2904 \\
2864 \\
2798 \\
-3 \%\end{array}$ & $<0.01$ & $\begin{array}{l}2926 \\
2868 \\
2866 \\
2774 \\
-5 \%\end{array}$ & $<0.01$ & $\begin{array}{l}2876 \\
2900 \\
2874 \\
2828 \\
-2 \%\end{array}$ & $0 \cdot 1$ & $<0.01$ & $0 \cdot 15$ \\
\hline
\end{tabular}

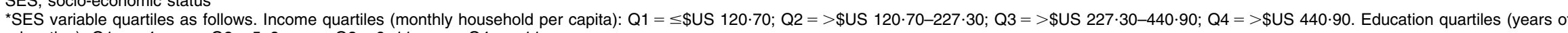
education): Q1 = $\leq 4$ years; $22=5-8$ years; $\mathrm{Q}=9-11$ years; $\mathrm{Q} 4=>11$ years.

tEighty-five women were excluded because they had no information about education.

IIFurther adjusted for energy intake as a continuous variable by a linear model.

Calculated as retinol activity equivalents. 

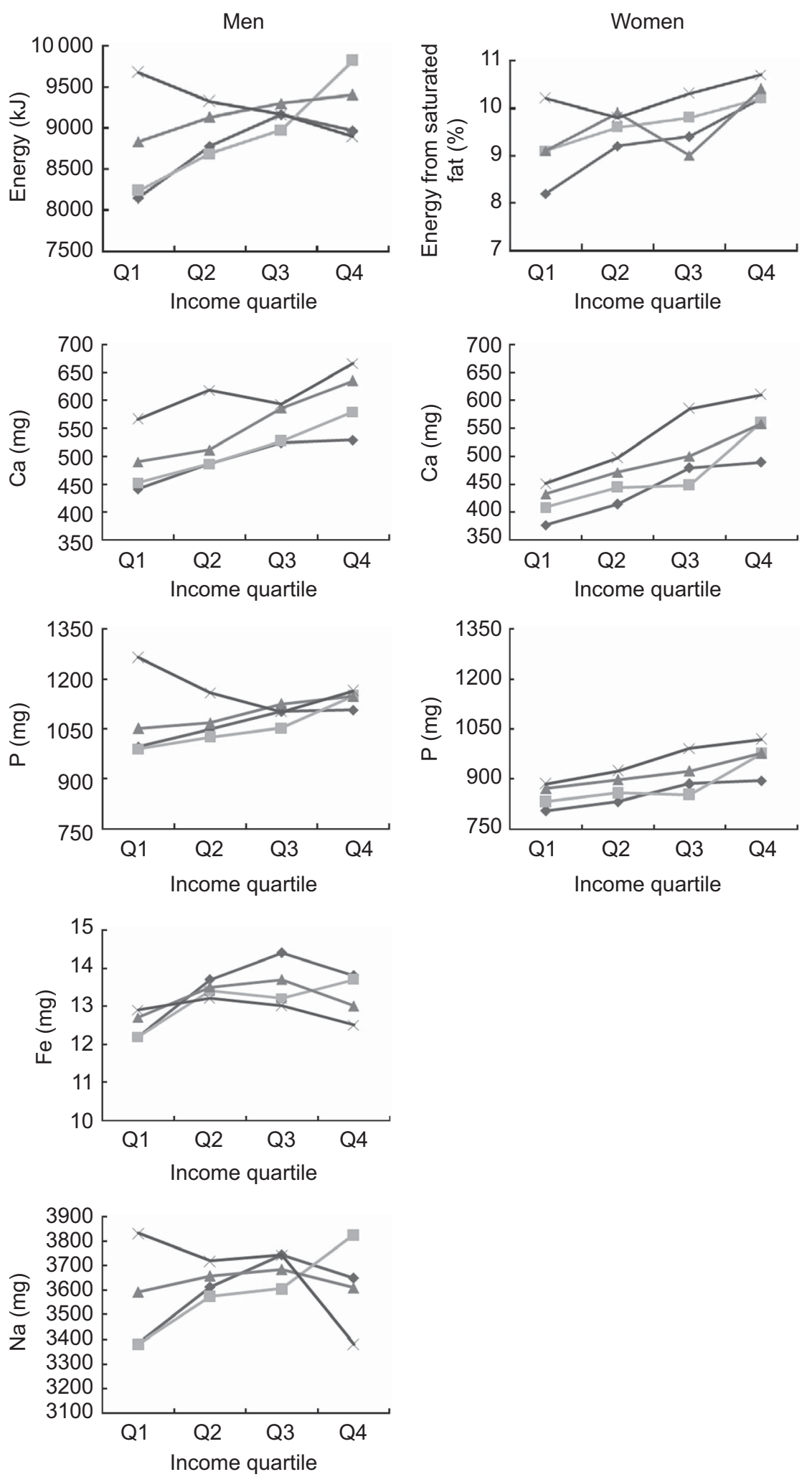

Fig. 1 Aged-adjusted weighted mean nutrient intakes according to quartiles of both income and education for those nutrients with a statistically significant interaction term between income and education according to sex; Brazilian adults aged 20-59 years, 9893 men and 10944 women (excluding pregnant and lactating women), 2008-2009 National Dietary Survey. Income quartiles (monthly household per capita): Q1 = S\$US 120.70; Q2 =>\$US 120.70-227.30; Q3 =>\$US 227.30-440.90; Q4 =>\$US 440.90.

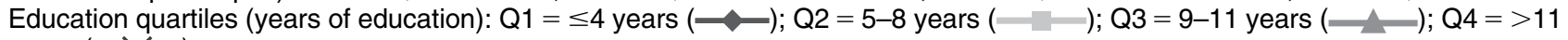
years $(\longrightarrow)$ 
with higher intakes of energy, sugar and sugar-sweetened beverages and lower dietary moderation. The authors concluded that immigrant women followed the traditional diet patterns of their native country.

One aspect to be highlighted is that the high prevalences of inadequate nutrient intakes observed in the present study remained so across all income and education quartiles, despite the negative association with these variables. This finding is particularly important from a public health perspective, considering that Brazilian adults could be at risk of several diseases caused by both deficiencies in micronutrient intake (particularly Ca deficiency, vitamin $\mathrm{A}$ and $\mathrm{C}$ inadequacy) and excessive nutrient intake (especially saturated fat and $\mathrm{Na}$ intakes).

An interesting finding was the role of education in $\mathrm{Ca}$ and $\mathrm{Na}$ intakes in both sexes. Ca intake in both sexes was associated only with the interaction term of income and education in mutually adjusted models. This means that education intensified the association between income and $\mathrm{Ca}$ intake among women, in which the increase of $\mathrm{Ca}$ intake with income was much more evident among people in the highest educational level. The same was verified for $\mathrm{P}$ intake among women. Nevertheless, the behaviour of $\mathrm{Ca}$ intake among men in the highest educational level was modified by income, with a decrease of $\mathrm{Ca}$ intake from the second to the third income quartiles. Moreover, education modified the association between income and $\mathrm{Na}$ intake among men. We observed a positive association between income and $\mathrm{Na}$ intake among men from all educational levels with the exception of men from the highest educational level, for which $\mathrm{Na}$ intake decreased with income. $\mathrm{Na}$ intake among women was also associated only with education in mutually adjusted models. Similar results about the role of education were found in the USA, in that education had a stronger effect on diet quality than did income ${ }^{(4,19)}$.

It is relevant to mention the difference between sexes in the association of socio-economic variables and $\mathrm{Fe}$ intake. While education had the main role on Fe intake among women, only income was associated with Fe consumption among men. Hence, the strategy to increase Fe consumption, mainly among women who are the more vulnerable group to inadequacy, may be dependent on educational level improvement. Furthermore, vitamin $\mathrm{B}_{12}$ intake was associated only with education among women, reaffirming that to reach a better diet quality among women it is fundamental to enhance educational level.

On the other hand, diet cost has been shown to be a mediator of the relationship between SES and diet quality. Aggarwal et $a l^{(16)}$ found that socio-economic disparities in diet quality may be explained by differential diet cost. The authors concluded that diet cost is one of the critical factors that may explain the consumption of a lower-quality diet among individuals in the lower socio-economic strata, while other studies demonstrated that diet quality was positively associated with diet cost ${ }^{(9,10,44-46)}$. Nevertheless, diet cost does not explain all of our results considering that the intakes of energy and energy from saturated fat were positively associated with income in both sexes and dietary fibre intake was negatively associated with income status. They were all markers of lower diet quality in highestincome group.

The high prevalences of inadequate nutrient intakes observed could be associated with underestimation of food intake. Although we chose not to exclude underor over-reporting in our sample, the estimate of usual nutrient intake was done using the NCI method and MSM, which adjust for within-person variance, correcting extreme and unlikely intake (both related to under- or overestimates) ${ }^{(47)}$. In addition, the study design allowed for analysing food consumption over 1 year, which captures the seasonal variation of Brazilian food habits.

A possible limitation of the present study was that it did not consider vitamin and mineral supplemental intake. A Canadian study showed that the prevalence of supplement use was significantly higher among high-income households and individuals with a higher education level ${ }^{(48)}$. Although we did not estimate vitamin and mineral supplementation, we are of the opinion that this may not change our results because the use of supplements in Brazil is low and is usually recommended for specific groups, such as pregnant women ${ }^{(49)}$ and athletes ${ }^{(50)}$.

The present population-based study suggests that the role of income and education are independent and their meanings differ according to sex and the nutrient analysed. Purchasing capability alone does not necessarily lead to a healthy diet, as well as a higher education level does not result always in better food choices. Education is one important step to improve nutrient intake in Brazil; emphasis should be laid on enhancing dietary knowledge and formulating economic strategies that would allow lower-income individuals to adopt a healthy diet.

\section{Acknowledgements}

Sources of funding: The survey was funded by the Ministry of Health of Brazil (agreement number 4652/2005; grant number $25000.202044 / 2005-16$ ). The funder contributed to the study design and conduct of the study. M.C.A. received a fellowship from the Brazilian Federal Agency for the Improvement of Higher Education (Coordenação de Aperfeiçoamento de Pessoal de Nível Superior; CAPES). Conflicts of interest: None of the authors has conflicts of interest. Authors' contributions: M.C.A. contributed to the analysis and interpretation of the data and the drafting of the manuscript; E.V.J. contributed to the analysis and interpretation of the data; W.L.J. contributed to the interpretation of the data; R.S. contributed to the design of the study, analysis and interpretation of the data and the drafting of the manuscript. All authors read and approved the final manuscript. 


\section{References}

1. Irala-Estevez JD, Groth M, Johansson L et al. (2000) A systematic review of socio-economic differences in food habits in Europe: consumption of fruit and vegetables. Eur J Clin Nutr 54, 706-714.

2. Kant AK \& Graubard BI (2007) Secular trends in the association of socio-economic position with self-reported dietary attributes and biomarkers in the US population: National Health and Nutrition Examination Survey (NHANES) 1971-1975 to NHANES 1999-2002. Public Health Nutr 10, 158-167.

3. Darmon N \& Drewnowski A (2008) Does social class predict diet quality? Am J Clin Nutr 87, 1107-1117.

4. Monsivais P \& Drewnowski A (2009) Lower-energy-density diets are associated with higher monetary costs per kilocalorie and are consumed by women of higher socioeconomic status. J Am Diet Assoc 109, 814-822.

5. Mullie P, Clarys P, Hulens M et al. (2010) Dietary patterns and socioeconomic position. Eur J Clin Nutr 64, 231-238.

6. Kim K, Hong SA \& Kim MK (2010) Trends in nutritional inequality by educational level: a case of South Korea. Nutrition 26, 791-798.

7. Hopping BN, Erber E, Mead E et al. (2010) Socioeconomic indicators and frequency of traditional food, junk food, and fruit and vegetable consumption amongst Inuit adults in the Canadian Arctic. J Hum Nutr Diet 23, Suppl. 1, S51-S58.

8. Wang Y \& Chen X (2011) How much of racial/ethnic disparities in dietary intakes, exercise, and weight status can be explained by nutrition- and health-related psychosocial factors and socioeconomic status among US adults? J Am Diet Assoc 111, 1904-1911.

9. Rehm CD, Monsivais P \& Drewnowski A (2011) The quality and monetary value of diets consumed by adults in the United States. Am J Clin Nutr 94, 1333-1339.

10. Iannotti LL, Robles M, Pachón H et al. (2012) Food prices and poverty negatively affect micronutrient intakes in Guatemala. J Nutr 142, 1568-1576.

11. Alkerwi A, Sauvageot N, Nau A et al. (2012) Population compliance with national dietary recommendations and its determinants: findings from the ORISCAV-LUX study. $\mathrm{Br} \mathrm{J}$ Nutr 108, 2083-2092.

12. Kirkpatrick SI, Dodd KW, Reedy J et al. (2012) Income and race/ethnicity are associated with adherence to food-based dietary guidance among US adults and children. I Acad Nutr Diet 112, 624-635.

13. Aggarwal A, Monsivais P \& Drewnowski A (2012) Nutrient intakes linked to better health outcomes are associated with higher diet costs in the US. PLOSONE 7, e37533.

14. Monteiro CA, Conde WL \& Popkin BM (2007) Incomespecific trends in obesity in Brazil: 1975-2003. Am J Public Health 97, 1808-1812.

15. da Fonseca M de J, Faerstain E \& Chor D et al. (2006) Associations between schooling, income, and body mass index among public employees at an university in Rio de Janeiro, Brazil: the Pró-Saúde Study. Cad Saude Publica 22, 2359-2367.

16. Aggarwal A, Monsivais P, Cook AJ et al. (2011) Does diet cost mediate the relation between socioeconomic position and diet quality? Eur J Clin Nutr 65, 1059-1066.

17. Turrell G, Hewitt B, Patterson C et al. (2003) Measuring socio-economic position in dietary research: is choice of socio-economic indicator important? Public Health Nutr $\mathbf{6}$, 191-200.

18. Féart C, Jutand MA, Larrieu S et al. (2007) Energy, macronutrient and fatty acid intake of French elderly community dwellers and association with socio-demographic characteristics: data from the Bordeaux sample of the Three-City Study. Br J Nutr 98, 1046-1057.
19. Drewnowski A \& Specter SE (2004) Poverty and obesity: the role of energy density and energy costs. Am J Clin Nutr 79, 6-16.

20. Instituto Brasileiro de Geografia e Estatística (2009) Pesquisa Nacional por Amostra de Domicilios (National Household Sample Survey). Rio de Janeiro: IBGE, Ministério do Planejamento, Orçamento e Gestão.

21. Araujo MC, Bezerra IN, Barbosa F dos S et al. (2013) Macronutrient consumption and inadequate micronutrient intake in adults. Rev Saude Publica 47, Suppl. 1, 177S-189S.

22. Instituto Brasileiro de Geografia e Estatística (2011) Pesquisa de Orçamentos Familiares, 2008-2009. Análise do Consumo Alimentar Pessoal no Brasil (Household Expenditure Survey 2008-2009: Analysis of the Personal Food Consumption in Brazil). Rio de Janeiro: IBGE, Ministério do Planejamento, Orçamento e Gestão.

23. Instituto Brasileiro de Geografia e Estatística (2011) Pesquisa de Orçamentos Familiares 2008-2009: Tabelas de Composição Nutricional de Alimentos Consumidos no Brasil (Household Expenditure Survey 2008-2009: Tables of Nutritional Composition of Food Consumed in Brazil). Rio de Janeiro: IBGE, Ministério do Planejamento, Orçamento e Gestão.

24. Instituto Brasileiro de Geografia e Estatística (2011) Pesquisa de Orçamentos Familiares 2008-2009: Tabela de Medidas Referidas para os Alimentos Consumidos no Brasil (Household Expenditure Survey 2008-2009: Table of Reference Measures for Food Consumed in Brazil). Rio de Janeiro: IBGE, Ministério do Planejamento, Orçamento e Gestão.

25. Tooze JA, Midthune D, Dodd KW et al. (2006) A new statistical method for estimating the usual intake of episodically consumed foods with application to their distribution. J Am Diet Assoc 106, 1575-1587.

26. Freedman LS, Guenther PM, Dodd KW et al. (2010) The population distribution of ratios of usual intakes of dietary components that are consumed every day can be estimated from repeated 24-hour recalls. J Nutr 140, 111-116.

27. Fay RE (1989) Theory and application of replicate weighting for variance calculations. Proceedings of the Survey Research Methods Section, American Statistical Association 212-217.

28. Barbosa F, dos S, Sichieri R \& Junger WL (2013) Assessing usual dietary intake in complex sample design surveys. Rev Saude Publica 47, Suppl. 1, 171S-176S.

29. Kish L \& Frankel MR (1968) Balanced repeated replication for analytical statistics. Proceedings of the Social Statistics Section, American Statistical Association 2-11.

30. Institute of Medicine, Food and Nutrition Board (1997) Dietary Reference Intakes for Calcium, Phosphorus, Magnesium, Vitamin D, and Fluoride. Washington, DC: National Academy Press.

31. Institute of Medicine, Food and Nutrition Board (1998) Dietary Reference Intakes for Thiamin, Riboflavin, Niacin, Vitamin $B_{6}$, Folate, Vitamin $B_{12}$, Pantothenic Acid, Biotin, and Choline. Washington, DC: National Academy Press.

32. Institute of Medicine, Food and Nutrition Board (2000) Dietary Reference Intakes for Vitamin C, Vitamin E, Selenium, and Carotenoids. Washington, DC: National Academy Press.

33. Institute of Medicine, Food and Nutrition Board (2001) Dietary Reference Intakes for Vitamin A, Vitamin $K$, Arsenic, Boron, Chromium, Copper, Iodine, Iron, Manganese, Molybdenum, Nickel, Silicon, Vanadium, and Zinc. Washington, DC: National Academy Press.

34. Institute of Medicine, Food and Nutrition Board (2004) Dietary Reference Intakes for Water, Potassium, Sodium, Chloride, Sulfate. Washington, DC: National Academy Press. 
35. Institute of Medicine, Food and Nutrition Board (2010) Dietary Reference Intakes for Calcium and Vitamin D. Washington, DC: National Academy Press.

36. Beaton GH (1994) Criteria of an adequate diet. In Modern Nutrition in Health and Disease, 8th ed., pp. 1491-1505 [ME Shils, JA Olson and M Shike, editors]. Philadelphia, PA: Lea \& Febiger.

37. Sarno F, Claro RM, Levy RB et al. (2009) Estimated sodium intake by the Brazilian population, 2002-2003. Rev Saude Publica 43, 219-224.

38. Sposito AC, Caramelli B, Fonseca FA et al. (2007) IV Brazilian Guideline for Dyslipidemia and Atherosclerosis prevention: Department of Atherosclerosis of Brazilian Society of Cardiology. Arq Bras Cardiol 88, Suppl. 1, S2-S19.

39. Brasil Ministério da Saúde, Secretaria Nacional de Assistência à Saúde, Departamento de Atenção Básica, Coordenação Geral da Política de Alimentação e Nutrição (2006) Guia alimentar para a população brasileira: promovendo a alimentação saudável. Brasília: Ministério da Saúde.

40. Harttig U, Haubrock J, Knuppel J et al. (2011) The MSM program: web-based statistics package for estimating usual dietary intake using the Multiple Source Method. Eur J Clin Nutr 65, Suppl. 1, S87-S91.

41. Souza A de M, Pereira RA, Yokoo EM et al. (2013) Most consumed foods in Brazil: National Dietary Survey 2008-2009. Rev Saude Publica 47, Suppl. 1, 190S-199S.

42. Bezerra IN, Souza A de M, Pereira RA et al. (2013) Contribution of foods consumed away from home to energy intake in Brazilian urban areas: the 2008-9 Nationwide Dietary Survey. Br J Nutr 109, 1276-1283.

43. Tseng M \& Fang CY (2012) Socio-economic position and lower dietary moderation among Chinese immigrant women in the USA. Public Health Nutr 15, 415-423.

44. Drewnowski A, Darmon N \& Briend A (2004) Replacing fats and sweets with vegetables and fruits - a question of cost. Am J Public Health 94, 1555-1559.

45. Drewnowski A \& Darmon N (2005) Food choices and diet costs: an economic analysis. J Nutr 135, 900-904.

46. Appelhans BM, Milliron BJ, Woolf $\mathrm{K}$ et al. (2012) Socioeconomic status, energy cost, and nutrient content of supermarket food purchases. Am J Prev Med 42, 398-402.

47. American Dietetic Association (2011) Practice paper of the American Dietetic Association: using the Dietary Reference Intakes. J Am Diet Assoc 111, 762-770.

48. Vatanparast H, Adolphe JL \& Whiting SJ (2010) Socioeconomic status and vitamin/mineral supplement use in Canada. Health Rep 21, 1-7.

49. Rondó PHC, Fukushima CM \& Moraes F (2006) Vitaminmineral supplement use by low-income Brazilian pregnant adolescents and non-adolescents and the predictors for non-use. Eur J Clin Nutr 60, 1108-1114.

50. Pereira RF, Lajolo FM \& Hirschbruch MD (2003) Consumo de suplementos por alunos de academias de ginástica em São Paulo. Rev Nutr 16, 265-272. 